\title{
Micromechanical Modeling for Material Design of Durable Infrastructural Materials: The Influence of Aggregate and Matrix Modification on Elastic Behavior of Mortars
}

\author{
Sumanta Das, Amit Maroli, and Narayanan Neithalath \\ School of Sustainable Engineering and the Built Environment, Arizona State University, Tempe, AZ, USA
}

\begin{abstract}
This paper reports the fundamental difference in microstress distributions in traditional hardened cement paste with quartz inclusions and a cement paste with lightweight aggregate (LWA) inclusions using microstructurebased numerical simulation involving finite element method with periodic boundary conditions. Variation of relative stress distributions under varying component material properties and varying microstructural features in both the systems is elucidated for a comprehensive understanding. The presented microstructure-based numerical technique accurately captures the stress concentrations inside microheterogeneous systems, which is otherwise not detectible using analytical homogenization schemes. Numerical simulations reveal that strengthening and stiffening of matrix and interfacial transition zone (ITZ) with silica fume incorporation as cement replacement in LWA incorporated cementitious systems has a detrimental effect in terms of overall strength of the material, contrary to traditional quartz-based cement mortar system. Proper selection of stiffness and strength of LWA inclusions is critical towards performance of such materials. This paper links the microstructure with mechanical behavior of two different microheterogeneous materials and provides valuable input towards material design of such non-traditional cementitious systems with different inclusions.
\end{abstract}

\section{INTRODUCTION}

The link between the microstructure and mechanical property provides valuable information towards design and development of composites for engineering applications. Cementations materials are the most widely used composite material in this world and their performance is critical in structural installations such as high rise buildings, bridges, and nuclear plants. In the recent years, traditional concrete is being modified by using supplementary cementations materials as cement replacement or completely alternative cement-free binders are proposed for reduction in carbon footprint (Das, Souliman, Stone, \& Neithalath, 2014; Lothenbach, Scrivener, \& Hooton, 2011; Ravikumar, Peethamparan, \& Neithalath, 2010). Besides, the traditional quartz inclusions are being replaced by lightweight aggregates (Al-Jabri, Hago, Al-Nuaimi, \& Al-Saidy, 2005; Cusson \& Hoogeveen, 2008; Kim, Jeon, \& Lee, 2012; Nguyen, Beaucour, Ortola, \& Noumowé, 2014) for various benefits such as reduction of dead load, and thermal and acoustic insulation. Modification in the matrix/inclusion is likely to have a significant influence on the stress distribution in the microstructure and thus on the failure path/mechanism of the material. Therefore, a comprehensive understanding of the influence of such modification on the microstress distribution in the microstructure is necessary in order to design such materials for optimal mechanical performance.
The current study focuses on understanding the fundamental difference in stress distribution in traditional cement mortar and lightweight aggregate mortar systems. The difference in stiffness between harder quartz particles and softer light weight aggregate inclusions is likely to impart a significant influence on the fundamental stress distribution characteristics in the mortar system, which has been studied in detail in this paperthrough micromechanical modelling. Researchers have studied the failure mechanisms of traditional concrete and lightweight aggregate concrete (LWAC) by employing experiment-based empirical formulations (Ke, Ortola, Beaucour, \& Dumontet, 2014) and through micromechanical modelling involving analytical techniques such as Mori-Tanaka approach (Nilsen, Monteiro, \& Gjørv, 1995; Yang, 1997; Yang \& Huang, 1998), double inclusion model (Stora, He, \& Bary, 2006; Yang \& Huang, 1996) and iterative homogenization technique (Das et al., 2015; Dunant et al., 2013). Analytical homogenization techniques have been shown to provide good estimates. However, application of these techniques has been shown to be limited if the phase stiffness contrast is too high or the morphology is very complicated. Besides, these analytical and semi-analytical homogenization techniques do not accurately predict local stress concentrations around inclusions, which influence macroscopic behavior especially for microheterogeneous materials. Thus, numerical modelling using microstructural 
representative volume element (RVE) is a better approach under such scenarios. In this paper, 2D virtual periodic microstructures for quartz mortar and LWA mortar systems with spherical inclusions including interfacial transition zone (ITZ) around inclusions are generated virtually and the representative element areas (REA), thus obtained, are numerically analyzed using finite element simulation involving periodic boundary conditions ( $\mathrm{Li}, 2008$; Sluis, Schreurs, Brekelmans, \& Meijer, 2000; Xia, Zhou, Yong, \& Wang, 2006). Stresses, thus obtained in REA and individual microstructural components, are evaluated to bring out fundamental difference in stress distributions imparted by LWA inclusions over traditional quartz particle inclusions. In addition, the influence of matrix stiffening in both the systems is elucidated. Such a comprehensive numerical evaluation of fundamental difference in mechanical behavior or traditional quartz mortar and LWA mortar system has not been reported in the literature to the best of our knowledge. Such quantitative information on the fundamental difference in stress distributions and local stress concentrations within two fundamentally different microheterogeneous systems provides valuable indication on relative damage and debonding of the inclusions from the matrix, which is critical towards appropriate design and improvement of such materials.

\section{MATERIAL PROPERTIES AND MICROSTRUCTURAL MODELS}

The constitutive relationships for all the components: cement paste, hard (quartz aggregates), and soft (lightweight aggregate - LWA) inclusions, and the paste-inclusion interfaces are considered in their respective linear elastic regimes only.

The default elastic properties of the components, extracted from available literature (Grondin \& Matallah, 2014; Hashin \& Monteiro, 2002; Ke, Beaucour, Ortola, Dumontet, \& Cabrillac, 2009; Lutz, Monteiro, \& Zimmerman, 1997; Nilsen et al., 1995; Yang, 1998; Zanjani \& Bobko, 2014), are presented in Table 1. However, for parametric studies discussed later in the paper, a range of values are considered, which are indicated in the respective sections.

Table 1. Elastic properties of the components of the mortar for FE simulation.

\begin{tabular}{|c|c|c|c|c|c|}
\hline $\begin{array}{c}\text { Elastic } \\
\text { property }\end{array}$ & $\begin{array}{c}\text { Hardened } \\
\text { cement } \\
\text { paste }\end{array}$ & Quartz & $\begin{array}{c}\text { Quartz- } \\
\text { cement } \\
\text { paste } \\
\text { interface }\end{array}$ & LWA & $\begin{array}{l}\text { LWA- } \\
\text { cement } \\
\text { paste } \\
\text { interface }\end{array}$ \\
\hline $\begin{array}{l}\text { Young's } \\
\text { modulus (GPa) }\end{array}$ & 20 & 70 & 15 & 16 & 30 \\
\hline Poisson's ratio & 0.22 & 0.17 & 0.22 & 0.20 & 0.20 \\
\hline
\end{tabular}

Two-dimensional plain strain finite element models are employed here in order to characterize the influence of inclusion and matrix modifications on the bulk elastic behavior of the binder system. A sufficiently large $(4.15 \mathrm{~mm} \times 4.15 \mathrm{~mm})$ representative element area (REA) has been considered for analysis. The virtual random periodic microstructure is generated using a microstructural stochastic packing algorithm (Kumar et al., 2013; Meier, Kuhl, \& Steinmann, 2008; Torquato, 2013). This algorithm requires the particle size distribution (PSD) and the volume fraction of particles as inputs and it packs the circular inclusions with an interface layer of predefined thickness around them inside a REA of $4.15 \mathrm{~mm} \times 4.15 \mathrm{~mm}$. Generation of particles and their packing in the REA is allowed if the minimum distance between the centroids of neighboring particles is always greater than the sum of their radii, i.e., the interfacial zones are allowed to overlap in this packing scheme. Generation and random spatial placement of inclusions goes on simultaneously until the target volume fraction of inclusions is reached. The algorithm is described in detail in Meier et al. (2008). Figure 1(a) shows the generated periodic microstructure.

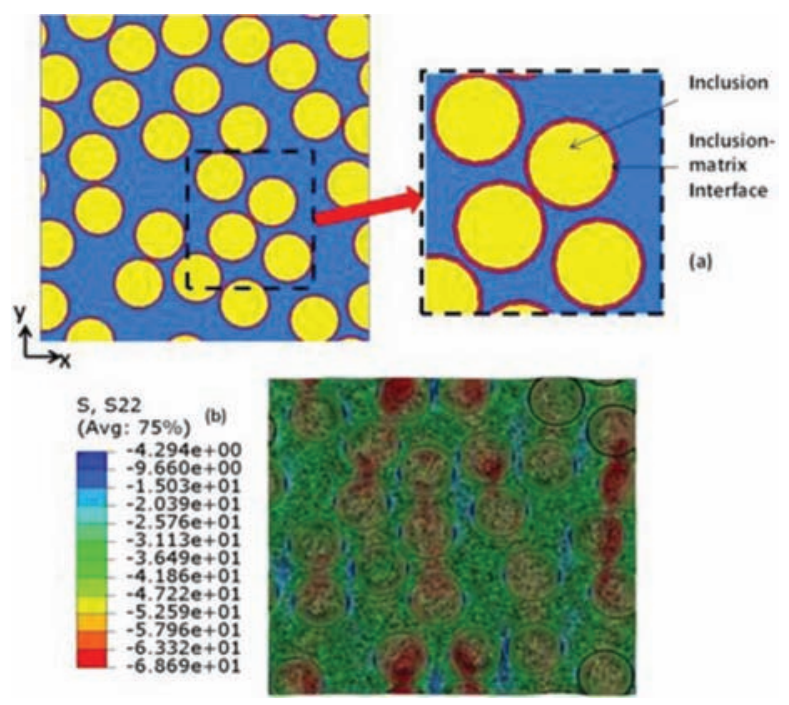

Figure 1. (a) FE model showing the inclusions with the interfacial zones around them. The model contains $50 \%$ of inclusions by volume (or area); (b) effect of random distribution and periodic boundary conditions on the stress distribution under an imposed strain of $0.12 \%$. The average REA stress is $36.13 \mathrm{MPa}$.

In order to eliminate the boundary effects, periodic boundary conditions (Li, 2008; Sluis et al., 2000; Xia et al., 2006) are employed in the 2-D REA, as shown in Figure 2. Figure 2(a) shows schematic periodic arrays of repetitive unit cells, and Figure 2(b) shows the periodic boundary conditions applied on one of such schematic representative elements for illustration. Periodic boundary condition ensures two continuity criteria at the boundaries of neighboring unit cells in order to ensure 
assembly of individual unit cells as a physical continuous body (Suquet, 1987): (i) displacement continuity, i.e., neighboring unit cells cannot be separated or they cannot penetrate each other, and (ii) traction continuity at the boundary of neighboring unit cells. The displacement field in any periodic microstructure is given as:

$$
v_{i}\left(x_{1}, x_{2}\right)=\varepsilon_{i j}^{0} x_{j}+v_{i}^{*}\left(x_{1}, x_{2}\right)
$$

here, $\varepsilon_{i j}^{0}$ is the applied strain tensor and $v_{i}^{*}$ is a periodic function representing the modification of linear displacement field due to the heterogeneous microstructure. For, the unit cell shown in Figure 2(b), the displacements on a pair of parallel opposite boundary edges are given as:

$$
\begin{aligned}
& v_{i}^{s+}=\varepsilon_{i j}^{0} x_{j}^{s+}+v_{i}^{*} \\
& v_{i}^{s-}=\varepsilon_{i j}^{0} x_{j}^{s-}+v_{i}^{*}
\end{aligned}
$$

here, $s^{+}$and $s^{-}$are sth pair of two opposite parallel boundary surfaces of the unit cell, as shown in Figure 2(b). The periodic function $v^{*}$ is the same at both the parallel opposite edges due to periodicity. The difference between the displacement fields of the two opposite parallel boundary edges is given as:

$$
v_{i}^{s+}-v_{i}^{s-}=\varepsilon_{i j}^{0}\left(x_{j}^{s+}-x_{j}^{s-}\right)=\varepsilon_{i j}^{0} \Delta x_{j}^{s}
$$

For a pair of opposite parallel boundary edges, $\Delta x_{j}^{s}$ is constant for a specified $\varepsilon_{i j}^{0}$. Such equations are applied as nodal displacement constraints in the finite element (FE) microstructural analysis.

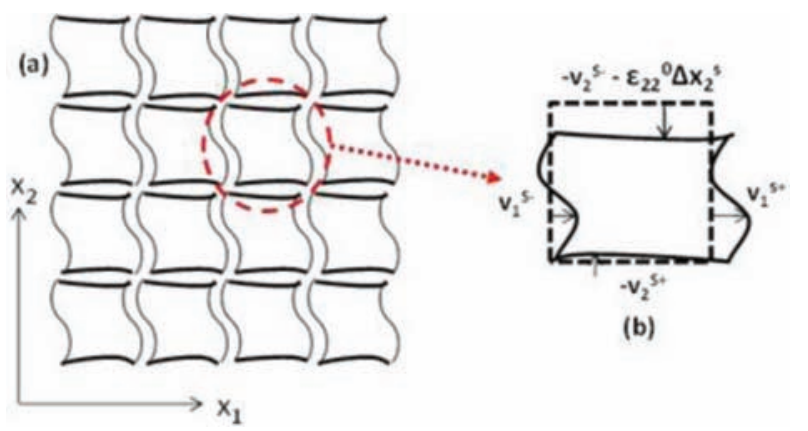

Figure 2. Schematic representative element area (REA) under applied strain $\left(\varepsilon_{22}^{0}\right)$ with periodic boundary conditions.

Periodic boundary condition is implemented on the REA as nodal displacement constraints through a Python language program appended to the orphan mesh file containing the periodic microstructure information. The REA is meshed using a Python script (Van Rossum, 2007) through ABAQUS ${ }^{\mathrm{TM}}$. A specific strain is applied on the REA, and the analysis is implemented through ABAQUS ${ }^{\mathrm{TM}}$ solver. Thus, this approach simulates a strain (or displacement)-controlled test scenario. To efficiently handle post-processing of the simulated individual element stresses, a homogenization module is developed to obtain effective area-averaged REA stresses/strains (Sun \& Vaidya, 1996) and the effective individual phase stresses/strains. Figure 1(b) shows the stress distribution obtained after analysis under the application of an imposed strain of $0.12 \%$ (which is well within the linear elastic range of cementitious systems).

\section{NUMERICAL RESULTS AND DISCUSSION}

This section evaluates sensitivity of averaged stress responses with varying material properties and geometric parameters in the microstructure and brings out the fundamental difference in elastic response between mortars prepared with quartz particles and lightweight aggregates. The virtual microstructures with random spatial placement of circular inclusions under periodic boundary conditions are used for numerical illustration. The size of inclusions is considered to be identical $(600 \mu \mathrm{m})$ for simplicity for both quartz and lightweight aggregate. However, the modelling technique and discussions presented herein are not restricted to such simplification and can tackle any realistic inclusion sizes. The effect of irregular inclusion size on the effective stresses is also demonstrated later in this paper. The thickness of inclusion-matrix interface has been kept constant at $30 \mu \mathrm{m}$. The default volume fraction of inclusion is $50 \%$. The default material properties of different components are reported in Table 1.

\subsection{Influence of inclusion stiffness}

Figures 3(a) and (b) show the dominant principal stress $\left(\sigma_{22}\right)$ distribution considering the material properties shown in Table 1 for both the quartz and LWA mortar systems, respectively, when a strain of $0.12 \%$ is applied to the REA. The LWA inclusions exhibit significantly higher deformation as compared to the quartz inclusions as can be seen from these figures, which is expected. While the quartz particles are highly stressed in the quartz mortar system, in the LWA mortar, it is the ITZ that bears the highest stress. This is expected considering the significantly higher stiffness of quartz particles as compared to that of LWA, as shown in Table 1. Another distinct observation in Figure 3 is that the magnitude of stress inside the quartz particles increases when the particles are very close to each other, attributable to the significant stiffness mismatch between the inclusions and the matrix. On the contrary, LWA mortar does not exhibit an increase in stress inside LWA. Instead, the stress concentration in the ITZ is higher if the neighboring particles are close to each other. Thus, the relative stiffness of the inclusions with respect to the matrix results in distinctively different stress distributions, and thus differing propensities of 
failure in the microstructure. Note that the magnitude of the maximum dominant principal stresses $\left(\sigma_{22}\right)$, which occur in the inclusions when the inclusion is stiffer and the inclusion-paste stiffness mismatch is higher, and in the ITZ when inclusion-paste stiffness mismatch is lower, are rather comparable. The quantified averaged REA stress and the stresses in the other microstructural phases are plotted in Figure 4 as a function of the inclusion stiffness.

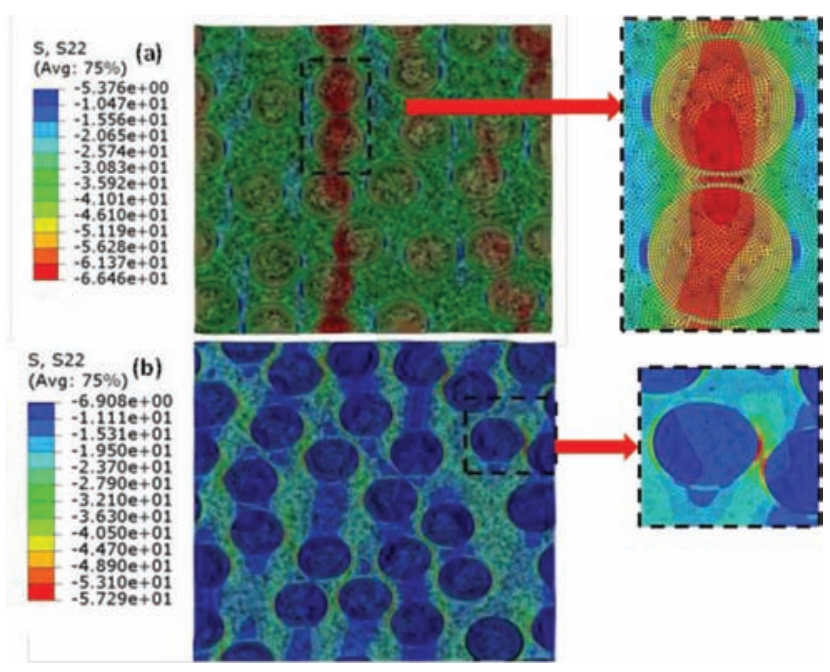

Figure 3. Dominant principal stress $\left(\sigma_{22}\right)(\mathrm{MPa})$ distributions in (a) quartz mortar system and (b) lightweight aggregate mortar system. Magnified representation of stress distributions in both mortar systems containing the zones around the inclusions are shown for clarity. The REA is subjected to a strain of $0.12 \%$.
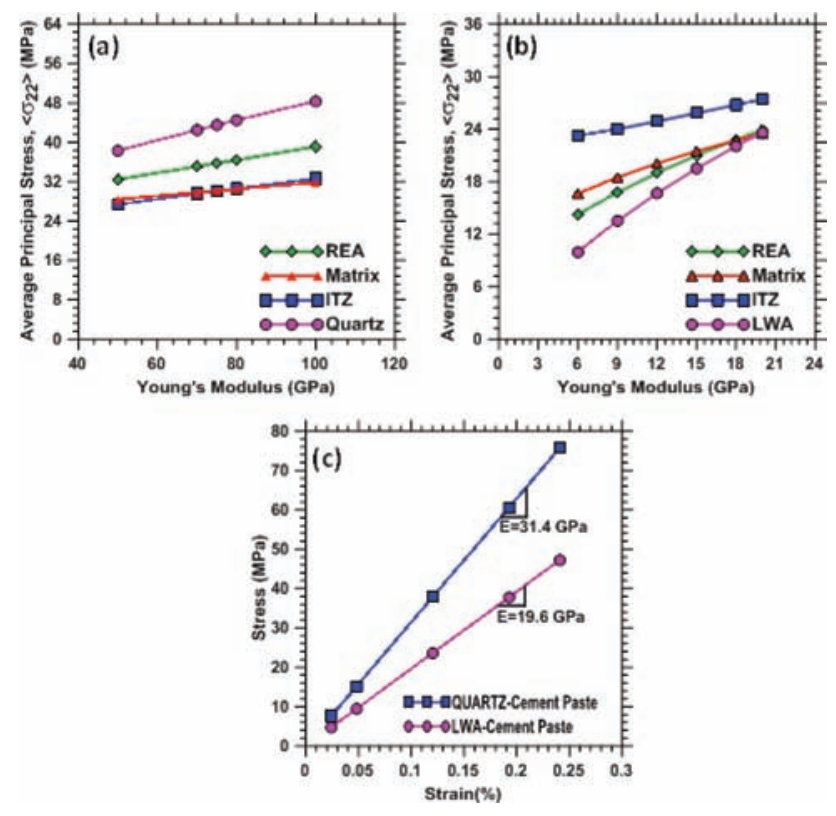

Figure 4. Effective REA and individual component stresses $\left(\sigma_{22}\right)$ as a function of inclusion stiffness for (a) quartz mortar system and (b) LWA mortar system; and (c) linear constitutive relationship for quartz and LWA mortar systems for defaults values of material parameters (shown in Table 1) and microstructural features.
Figure 4(a) shows the area-averaged dominant principal stresses in the REA and those in the individual microstructural phases for a mortar system containing quartz particles as a function of the Young's modulus of quartz (ranged from 50 to $100 \mathrm{GPa}$ ). With increasing $\mathrm{E}$ of quartz (at the same inclusion volume fraction, and leaving the $E$ of the paste and the interface unchanged), the average stresses in all the phases in the microstructure increase linearly. The quartz inclusions experience the highest average stress amongst all the phases because of the significantly higher $\mathrm{E}$ values compared to the paste or the ITZ, in line with Figure 3(a). The ITZ and the paste matrix components show similar averaged stresses, attributable to the fact that the stiffness difference between these phases is negligible when compared to the difference in stiffness between quartz and these phases.

Figure 4(b) shows the averaged REA and other component stresses in the LWA mortar system for varying stiffness of LWA inclusions [between 6 and $21 \mathrm{GPa}$ (Ke et al., 2009)]. Here, the highest stress is observed in the ITZ as shown in Figure 4(b) because of the fact that its stiffness is the highest among all the phases in this microstructure. While the average stress increases in all the phases when stiffer LWA is used, the rate of increase in stress is higher in the LWA inclusions compared to the other phases or the REA. The average stress in the cement paste matrix and ITZ of the LWA mortar system linearly increases with LWA stiffness, whereas the stress increase in the LWA inclusions is found to be nonlinear, primarily attributed to the deformational effects of the LWA and the consideration of perfect bonding between the phases in the model. The rate of stress increases in the LWA, the paste decreases with increasing LWA stiffness, and the stresses in these phases are almost equal when the LWA and the paste stiffness are similar, as expected. Figures 4(a) and (b) also show that the averaged stresses in all the components are lower in the LWA system as compared to those in quartz mortar system. This can be attributed to the stresses concentrated over large areas in quartz particles that increase the average inclusion stresses and the other phase stresses in quartz mortar system due to assumption of perfect bonding between phases. On the contrary, LWA system shows lower stresses due to lower stiffness of LWA inclusions and lower effective stiffness of REA.

Figure 4(c) shows the constitutive response of the quartz and LWA mortars containing $50 \%$ of inclusions by volume, extracted from numerical simulations. The dominant principal stresses $\left(\sigma_{22}\right)$ and principal strains $\left(\varepsilon_{22}\right)$ in the linear elastic range of these systems are used. Thus, the approach presented here can be used to determine the composite elastic moduli of systems 
containing multiple inclusion types. In addition to the $E$ value, as described earlier, this methodology also provides estimates of the microstresses in the different phases under imposed strains (in the linear elastic regime) and facilitates the development of constitutive relationships for composite materials, which otherwise would be experimentally tedious. Comparison of Young's modulus values obtained from numerical simulation (FEA) to those calculated using analytical/semi-analytical approaches and experimental validation of the adopted numerical technique is detailed in a later section of this paper.

\subsection{Influence of varying volume fraction of inclusion}

Figures 5(a) and (b) show the effect of volume fraction on the averaged dominant principal stress in quartz and LWA mortar systems, respectively.
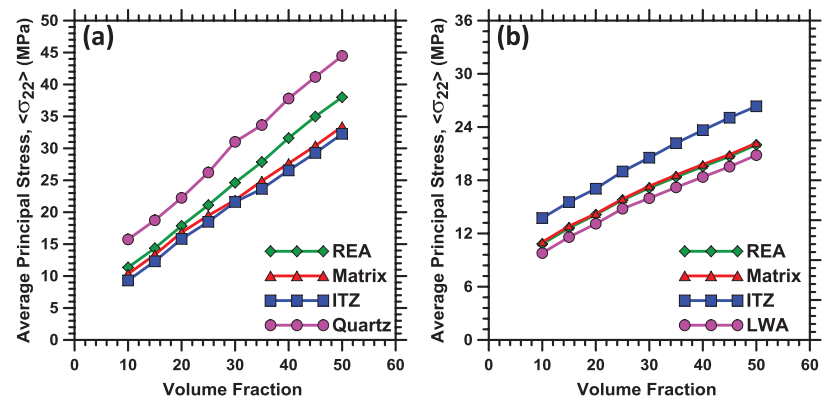

Figure 5. Effective REA and individual phase stresses as a function of inclusion volume fraction for (a) quartz mortar and (b) LWA mortar.

With increasing volume fraction of inclusion in the REA systems, the average stresses in all the components in both the model increase linearly. For the quartz model [Figure 5(a)], the rate of increase in component stresses with respect to increase in volume fraction is higher as compared to that in LWA mortar system [Figure 5(b)]. This is attributed to the higher stiffness of inclusions in quartz mortar systems as compared to LWA system as explained elsewhere in this paper. While increase in ITZ stress at higher volume fraction of quartz is likely to be responsible for interface failure (ITZ is the weakest component in quartz mortar system) and thus material failure under smaller applied strains in quartz mortar system, increase in stress inside weaker LWA (weakest component) inclusions at higher volume fractions of LWA is expected to be the cause of failure of LWA mortar system under smaller external applied strains.

\subsection{Influence of matrix and ITZ stiffening with silica fume addition}

This section reports the influence of matrix and ITZ strengthening/stiffening (including those accomplished through the use of additives such as silica fume as a partial replacement of cement) in mortars containing quartz or LWA as inclusions with an aim of understanding the relative influences of matrix modification. The Young's modulus of silica fume modified cement paste is taken as $25 \mathrm{GPa}$ (Hu \& $\mathrm{Li}, 2015)$ as opposed to $20 \mathrm{GPa}$ for the unmodified systems. The stiffness of the ITZ was also increased proportionally (Young's modulus of ITZ is taken as 18.75 and $37.5 \mathrm{GPa}$ in quartz and LWA mortars, respectively) since silica fume incorporation is known to result in interface densification and stiffening (Duan, Shui, Chen, \& Shen, 2013; Hu \& Li, 2015). Figures 6(a) and (b) show average stresses in the REA and in the individual microstructural phases corresponding to an applied strain of $0.12 \%$ for the quartz and LWA mortars.
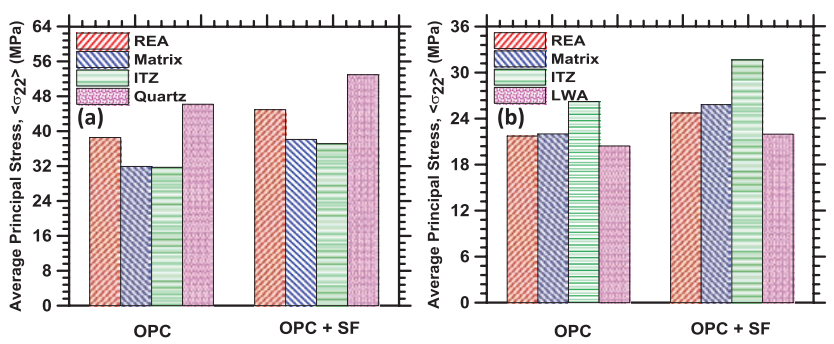

Figure 6. Effect of silica fume incorporation on the average REA and phase stresses for (a) mortar containing quartz inclusion and (b) mortar with LWA inclusions. The modified matrices contain $10 \%$ silica fume by mass as a cement replacement material.

Stresses increase in all the phases for both the mortar types when the paste phase contains silica fume. In the quartz mortar, the average stress in the ITZ increased by about $15 \%$ when $10 \%$ silica fume was incorporated. However, the strength enhancement of both the ITZ and the paste phase will likely be larger than the stress increase, thereby rendering improved mechanical performance to the quartz mortar when silica fume is incorporated in the paste phase. This has been demonstrated through experimental studies $\mathrm{Hu}$ \& Li, 2015; Shannag, 2000). The stress increase inside the quartz inclusions has an insignificant influence on material failure because of the higher strength of quartz particles (Axelson \& Edgar, 1950). On the contrary, the inclusions in the LWA mortar system are relatively weak and even a marginal increase in inclusion stress is likely to result in material failure at even lower applied strains as compared to that in LWA mortar systems without silica fume incorporation. Thus, the combined stiffening of ITZ and the matrix in LWA mortar system has a detrimental effect on the strength, provided it is the lightweight inclusion that is weaker and fails first. This point to the fact that matrix strengthening methods such as the use of silica fume might not be beneficial from a viewpoint of mechanical response in these systems, unless the LWA is stronger. However, the densification of the matrix and the ITZ will still lead to better durability properties in such concretes. 


\subsection{Influence of particle size distributions}

While all the previous parametric studies show response of single-sized inclusions, the current section shows the effect of irregular inclusion sizes on the stress response. Here, the ITZs are homogenized with the inclusions using analytical Mori-Tanaka approach (Mori \& Tanaka, 1973) as a compromise between computational efficiency and demand since generation of microstructure for a given particle size distribution with ITZ layer around inclusions is computationally expensive. Default values of material/ geometrical properties of inclusions and ITZ (Table 1) are used in homogenization process. Figures $7(\mathrm{a})$ and (b) show the dominant principal stress distribution in single sized and irregular inclusion sized LWA mortar system (mean $0.6 \mathrm{~mm}$ and standard deviation of $0.24 \mathrm{~mm}$ with uniform distribution), respectively. Figure 7(a) shows stress concentrations in between closely spaced single-sized LWA inclusions, especially if they are placed in the direction perpendicular to the direction of application of strain. On the other hand, Figure 7(b) shows a slight stress-relaxation trend in the LWA mortar system with non-uniform inclusion sizes compared to the single-sized case. Here also, the similar sized inclusions when placed in the direction perpendicular to the direction of application of strain show maximum stress concentrations. But, such higher stress concentrations are absent when the sizes of such inclusions are significantly different. These are reflected in the averaged dominant principal stresses in REA for various particle size distributions and individual components reported in Figure 8. In order to elucidate the effect of particle size distribution (PSD) on the quantified averaged stress

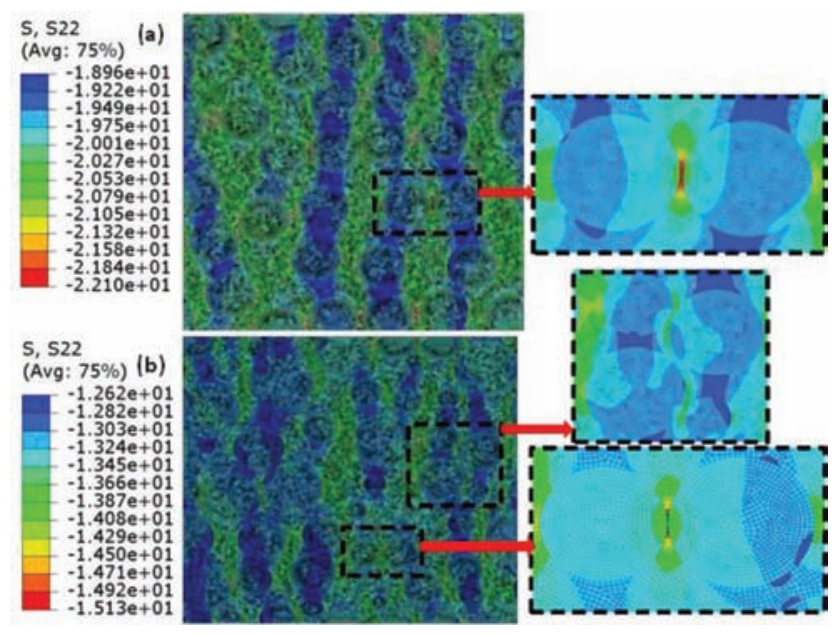

Figure 7. Influence of particle sizes on the dominant principal stresses (MPa) in the REA for (a) single-sized LWA inclusions and (b) multiple-sized $($ mean $=0.6 \mathrm{~mm}$ and standard deviation $=0.24 \mathrm{~mm}$ ) LWA inclusions embedded in a cement paste. Magnified representation of stress distributions in the vicinity of the particles (similar and dissimilar sizes) are shown for clarity. responses, three different PSDs with same mean size $(0.6 \mathrm{~mm})$ and varying standard deviation with uniform distribution is considered.

Figures 8(a) and (b) show averaged component and REA stress responses, respectively, for various particle size ranges of LWA inclusions.
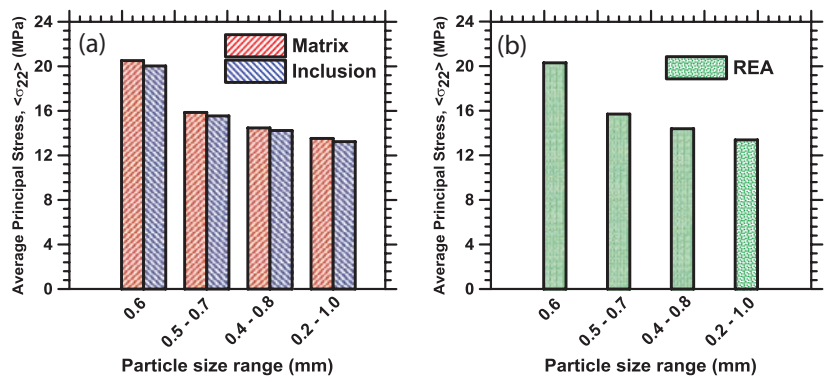

Figure 8. Effect of LWA inclusion size distribution on (a) the matrix and inclusion stresses and (b) effective REA stresses (mean particle size is $0.6 \mathrm{~mm}$ ).

The averaged stresses are highest in the single-sized LWA model due to higher stress concentrations in the inter-inclusion areas as explainer earlier. The stresses reduced considerably (by about 20\%) when nonuniform particle sizes are considered since interactions between neighboring smaller and larger particles show lower value of concentrated stresses, contrary to the higher stress concentrations encountered between two closely spaced similar-sized inclusions. For the same reason, the averaged stress in all components reduces as the standard deviation of particle size distribution is increased keeping the mean particle size same as reflected in Figures 8(a) and (b).

\section{CONCLUSION}

This paper employs a micromechanical model involving finite element analysis with periodic boundary conditions and reports the fundamental difference in stress distributions in traditional cement paste with quartz particle inclusions and lightweight aggregate mortar system. 2D periodic representative element areas (virtual microstructure) have been generated using a particle-packing based algorithm on known microstructural features. Periodic boundary conditions are applied on the 2-D REAs, and the microstructural stress analysis is performed using finite element method. The presented microstructurebased numerical technique accurately captures the stress concentrations inside microheterogeneous systems, which is otherwise not detectible using analytical homogenization schemes. Microstructural stress distribution and averaged REA stress as well as individual component stresses are evaluated under various parametric variations (material properties 
and microstructural features) in both the systems for a comprehensive understanding on their relative performance.

Numerical simulations reveal that traditional quartz mortar shows stress concentrations inside quartz particles, whereas LWA mortar system exhibits stress concentration at the LWA-cement paste interfaces due to significant difference in relative ITZ-inclusion stiffness in the two systems. While the failure of traditional quartz mortar system is not dictated by stress inside quartz particles due to their higher strength, the stress inside weaker LWA inclusions in LWA system becomes critical towards material failure due to lower strength of LWA inclusions. Hence, strengthening of LWA aggregates is a key to improve performance of such materials. But, increase in strength and thus stiffness of LWA inclusions results in increase in stress inside LWA inclusions. Hence, an optimal stiffness of LWA inclusions is desired with a perfect balance between increase in strength of LWA inclusions and associated stress increment.

Increase in stiffness of ITZ shows significant increase in all component stresses in quartz mortar system. On the contrary, such increase in ITZ stiffness results in stress increment only in ITZ in LWA system. The matrix/inclusion stresses in LWA system remain unaltered without any stress-relaxation inside LWA inclusions. Combined stiffening of matrix and ITZ increases in stresses, with techniques such as silica fume incorporation, as cement-replacement yields in all the components in both the systems. Increase in the ITZ stress (weakest component in quartz mortar system) can be balanced against enhancement of strength of ITZ and thus superior performance can be obtained through silica fume incorporation in traditional quartz mortar. But, same is not true for LWA system since the LWA stress increases with stiffness enhancement of ITZ and matrix while the strength of LWA remains unaltered under such scenario. Hence, strengthening and stiffening of matrix and ITZ are not a viable technique of quality improvement in LWA system, contrary to traditional quartz mortar system. This study links the microstructure with mechanical behavior of two different microheterogeneous materials to bring out their fundamental difference in microstress distribution and provides some valuable input towards material design of such non-traditional cementitious systems with different inclusions of varying stiffness.

\section{REFERENCES}

Al-Jabri, K. S., Hago, A. W., Al-Nuaimi, A. S., \& Al-Saidy, A. H. (2005). Concrete blocks for thermal insulation in hot climate. Cement and Concrete Research, 35(8), 1472-1479.
Axelson, J. W., \& Edgar, L. P. (1950). Crushing of single particles of crystalline quartz - Application of slow compression. Industrial \& Engineering Chemistry, 42(4), 665-670.

Cusson, D., \& Hoogeveen, T. (2008). Internal curing of high-performance concrete with pre-soaked fine lightweight aggregate for prevention of autogenous shrinkage cracking. Cement and Concrete Research, 38(6), 757-765.

Das, S., Souliman, B., Stone, D., \& Neithalath, N. (2014). Synthesis and properties of a novel structural binder utilizing the chemistry of iron carbonation. ACS Applied Materials \& Interfaces, 6(11), 8295-8304.

Das, S., Yang, P., Singh, S. S., Mertens, J. C. E., Xiao, X., Chawla, N., \& Neithalath, N. (2015). Effective properties of a fly ash geopolymer: Synergistic application of X-ray synchrotron tomography, nanoindentation, and homogenization models. Cement and Concrete Research, 78, 252-262.

Duan, P., Shui, Z., Chen, W., \& Shen, C. (2013). Effects of metakaolin, silica fume and slag on pore structure, interfacial transition zone and compressive strength of concrete. Construction and Building Materials, 44, 1-6.

Dunant, C. F., Bary, B., Giorla, A. B., Péniguel, C., Sanahuja, J., Toulemonde, J. C., ... Yvonnet, J. (2013). A critical comparison of several numerical methods for computing effective properties of highly heterogeneous materials. Advances in Engineering Software, 58, 1-12.

Grondin, F., \& Matallah, M. (2014). How to consider the interfacial transition zones in the finite element modelling of concrete? Cement and Concrete Research, 58, 67-75.

Hashin, Z., \& Monteiro, P. J. M. (2002). An inverse method to determine the elastic properties of the interphase between the aggregate and the cement paste. Cement and Concrete Research, 32(8), 1291-1300.

Hu, C., \& Li, Z. (2015). Property investigation of individual phases in cementitious composites containing silica fume and fly ash. Cement and Concrete Composites, 57, 17-26.

Ke, Y., Beaucour, A. L., Ortola, S., Dumontet, H., \& Cabrillac, R. (2009). Influence of volume fraction and characteristics of lightweight aggregates on the mechanical properties of concrete. Construction and Building Materials, 23(8), 2821-2828.

Ke, Y., Ortola, S., Beaucour, A. L., \& Dumontet, H. (2014). Micro-stress analysis and identification of lightweight aggregate's failure strength by micromechanical modeling. Mechanics of Materials, 68, 176-192.

Kim, H. K., Jeon, J. H., \& Lee, H. K. (2012). Workability, and mechanical, acoustic and thermal 
properties of lightweight aggregate concrete with a high volume of entrained air. Construction and Building Materials, 29, 193-200.

Kumar, A., Oey, T., Kim, S., Thomas, D., Badran, S., Li, J., ... Sant, G. (2013). Simple methods to estimate the influence of limestone fillers on reaction and property evolution in cementitious materials. Cement and Concrete Composites, 42, 20-29.

Li, S. (2008). Boundary conditions for unit cells from periodic microstructures and their implications. Composites Science and Technology, 68(9), 1962-1974.

Lothenbach, B., Scrivener, K., \& Hooton, R. D. (2011). Supplementary cementitious materials. Cement and Concrete Research, 41(12), 1244-1256. Conferences special: Cement hydration kinetics and modeling, Quebec City, 2009 \& CONMOD10, Lausanne, Switzerland, 2010.

Lutz, M. P., Monteiro, P. J. M., \& Zimmerman, R. W. (1997). Inhomogeneous interfacial transition zone model for the bulk modulus of mortar. Cement and Concrete Research, 27(7), 1113-1122.

Meier, H. A., Kuhl, E., \& Steinmann, P. (2008). A note on the generation of periodic granular microstructures based on grain size distributions. International Journal for Numerical and Analytical Methods in Geomechanics, 32(5), 509.

Mori, T., \& Tanaka, K. (1973). Average stress in matrix and average elastic energy of materials with misfitting inclusions. Acta Metallurgica, 21(5), 571-574.

Nguyen, L. H., Beaucour, A. L., Ortola, S., \& Noumowé, A. (2014). Influence of the volume fraction and the nature of fine lightweight aggregates on the thermal and mechanical properties of structural concrete. Construction and Building Materials, 51, 121-132.

Nilsen, A. U., Monteiro, P. J. M., \& Gjørv, O. E. (1995). Estimation of the elastic moduli of lightweight aggregate. Cement and Concrete Research, 25(2), 276-280.

Ravikumar, D., Peethamparan, S., \& Neithalath, N. (2010). Structure and strength of $\mathrm{NaOH}$ activated concretes containing fly ash or GGBFS as the sole binder. Cement and Concrete Composites, 32(6), 399-410.
Shannag, M. J. (2000). High strength concrete containing natural pozzolan and silica fume. Cement and Concrete Composites, 22(6), 399-406.

Sluis, V. O., Schreurs, P. J. G., Brekelmans, W. A. M., \& Meijer, H. E. H. (2000). Overall behaviour of heterogeneous elastoviscoplastic materials: Effect of microstructural modelling. Mechanics of Materials, 32(8), 449-462.

Stora, E., He, Q. C., \& Bary, B. (2006). Influence of inclusion shapes on the effective linear elastic properties of hardened cement pastes. Cement and Concrete Research, 36(7), 1330-1344.

Sun, C. T., \& Vaidya, R. S. (1996). Prediction of composite properties from a representative volume element. Composites Science and Technology, 56(2), 171-179.

Suquet, P. (1987). Elements of homogenization for inelastic solid mechanics.

Torquato, S. (2013). Random heterogeneous materials: Microstructure and macroscopic properties. Springer Science \& Business Media.

Van Rossum, G. (2007). Python programming language. USENIX annual technical conference (Vol. 41).

Xia, Z., Zhou, C., Yong, Q., \& Wang, X. (2006). On selection of repeated unit cell model and application of unified periodic boundary conditions in micro-mechanical analysis of composites. International Journal of Solids and Structures, 43(2), 266-278.

Yang, C. C. (1997). Approximate elastic moduli of lightweight aggregate. Cement and Concrete Research, 27(7), 1021-1030.

Yang, C. C. (1998). Effect of the transition zone on the elastic moduli of mortar. Cement and Concrete Research, 28(5), 727-736.

Yang, C. C., \& Huang, R. (1996). Double inclusion model for approximate elastic moduli of concrete material. Cement and Concrete Research, 26(1), 83-91.

Yang, C. C., \& Huang, R. (1998). Approximate strength of lightweight aggregate using micromechanics method. Advanced Cement Based Materials, 7(3-4), 133-138.

Zanjani, Z. V., \& Bobko, C. P. (2014). Nanomechanical characteristics of lightweight aggregate concrete containing supplementary cementitious materials exposed to elevated temperature. Construction and Building Materials, 51, 198-206. 